\title{
Sistemas inteligentes no diagnóstico da esquizofrenia
}

\author{
Denise Razzouk ${ }^{a}$, Itiro Shirakaw a,b, J air de J M aria \\ a'Departamento de Psiquiatria da Unifesp/EPM. 'Programa de Esquizofrenia (Proesq), Unifesp/EPM
}

\section{Sistemas inteligentes na psiquiatria}

O desenvolvimento de sistemas computadorizados inteligentes baseou-se, inicialmente, na premissa de que esses sistemas poderiam mimetizar comportamentos e raciocínios humanos. Posteriormente, em meados da década de 70, esses sistemas foram introduzidos na medicina por Shortliffe com o programa Mycin, que auxiliava na escolha de antibióticos. ${ }^{1}$

A partir dessa data, outros sistemas foram elaborados em diferentes domínios do conhecimento médico. Resultados de metanálises sobre a eficácia de tais sistemas apontam a evidência de que eles constituem ferramentas eficazes em diferentes procedimentos médicos, como no cálculo de dose de medicamentos, na escolha de uma terapêutica adequada e no reconhecimento de diagnósticos. ${ }^{2,3}$

A introdução desses sistemas na psiquiatria, a partir da década de 80 , também se direcionou para produzir sistemas que pudessem atuar como coadjuvantes na tomada de decisão clínica. No entanto, a avaliação crítica dos sistemas produzidos na área de saúde mental, bem como a evidência de seus benefícios para o uso clínico, ainda é insuficiente.

Em psiquiatria, a esquizofrenia constitui um transtorno de difícil diagnóstico, dada a sua característica de apresentação clínica heterogênea. Na última década, vários critérios diagnósticos operacionais foram desenvolvidos para identificar e classificar a esquizofrenia. $\mathrm{O}$ uso de critérios operacionais, no entanto, requer conhecimento e julgamento clínico que variam de acordo com a experiência do especialista.

Um dos objetivos de desenvolver um sistema diagnóstico de apoio à decisão é justamente aplicar o raciocínio heurístico empregado por um especialista na identificação de um diag- nóstico complexo. Dessa forma, o uso de tais sistemas na área da esquizofrenia pode facilitar a sua identificação.

Neste artigo serão abordados os sistemas de apoio à decisão no diagnóstico de esquizofrenia que foram desenvolvidos até o momento, suas vantagens, limitações e potenciais benefícios para a prática clínica.

\section{Definição e descrição de um sistema de apoio à decisão ou} "expert system"

"Expert systems”, ou sistemas de apoio à decisão, são programas capazes de reproduzir o raciocínio empregado na tomada de decisão de um especialista frente a um problema clínico, seja ele uma formulação de hipótese diagnóstica ou uma recomendação terapêutica. Esses programas são constituídos de uma base de conhecimento e de uma máquina inferencial.

O conhecimento pode ser oriundo de diferentes fontes. Pode ser proveniente da análise de dados, do conhecimento subjetivo baseado na experiência dos especialistas, de uma fonte de conhecimento teórico ou científico de um domínio específico e de estratégias de solução de problemas. ${ }^{4}$

As características principais de um "expert system" são: interação com o usuário por meio de linguagem natural; geração de hipóteses diagnósticas e explicação do raciocínio utilizado para cada conclusão; fornecimento de recomendações terapêuticas; emissão de alertas quanto a efeitos adversos de uma medicação, conduta medicamentosa inadequada, erros de prescrição e data para realizar exame.

Os programas inteligentes podem ser usados para selecionar um tratamento apropriado, levando em conta características individuais e os resultados das pesquisas. ${ }^{5}$ Esses sistemas podem propor

Tabela 1 - Vantagens e limitações dos "expert systems"

\section{Vantagens}

1. Auxilia o médico na tomada de decisão em ambiente clínico

2. Fornece informação atualizada, extensa e válida, de modo rápido e automatizado

3. Pode auxiliar no processo de aprendizado de reconhecimento de doenças para médicos iniciantes e não especialistas

4. Usado como um instrumento de polidiagnóstico para pesquisa

5. Permite que o médico possa avaliar suas condutas ao longo do tempo e comparar com um modelo teórico, diagnóstico ou um critério operacional
Limitações

1. A maioria dos sistemas não foi validada nem testada em ambiente clínico

2. Dificuldades com aceitação precisam ser minimizadas (interface com usuário)

3. A atualização da base de dados é cara, demorada e trabalhosa

4. Exige treinamento do usuário

5. Ausência de metodologia padronizada para seu desenvolvimento e avaliação 
cionar uma mudança no processo de obtenção de uma informação científica, uma vez que o meio de informação mais utilizado pelo médico é a consulta a um colega. ${ }^{6,7}$ No entanto, a informação obtida desse modo pode estar desatualizada e enviesada pela própria experiência do especialista, pois se baseia em uma população selecionada e não se aplica necessariamente a outros pacientes. $\mathrm{Na}$ Tabela 1 podem-se observar quais os benefícios que esses sistemas incrementam na prática clínica e as suas limitações.

\section{Sistemas inteligentes em esquizofrenia}

O Diagno foi um dos primeiros sistemas computacionais desenvolvido em psiquiatria pela equipe de Spitzer na década de $60 .{ }^{8}$ Esse sistema fornecia diagnósticos segundo os critérios da Associação Psiquiátrica Americana. Acoplado à segunda versão do Diagno, foi desenvolvido o programa PAU (Psychiatric Assessment Unit) por Thomas Williams et al, em 1974, para auxiliar no tratamento de pacientes psiquiátricos. ${ }^{9} \mathrm{O}$ impacto desse sistema em ambiente hospitalar em seis meses de uso produziu um decréscimo de $14 \%$ no tempo de hospitalização, um aumento de $48 \%$ na rotatividade e uma diminuição de $24 \%$ no número de retornos ao ambulatório.

Em 1978, Heiser \& Brooks desenvolveram o Headmed para auxiliar no tratamento de psicoses e de depressão, baseado em condutas empíricas de especialistas. ${ }^{1}$

Em 1987, surge o Psyxpert, desenvolvido no Texas por Overby et al e baseado nos critérios do DSM-III para o diagnóstico de transtornos psicóticos. ${ }^{1}$ Ainda nesse ano, Bronzino et al desenvolveram o Overseer, baseado em protocolos terapêuticos para transtornos afetivos bipolares, esquizofrenia e depressão maior. ${ }^{10}$ Esse sistema era capaz de emitir alertas quando as prescrições não correspondiam às recomendações dos protocolos e quando havia uma interação medicamentosa inadequada, e também relatava quanto tempo o paciente estava sem responder à medicação prescrita.

O DTREE foi desenvolvido em 1988 por Michael First et al em Nova York para auxiliar o clínico na identificação de diag- nósticos do eixo I do DSMIII-R. ${ }^{11} \mathrm{O}$ sistema está disponível no mercado, mas não há relatos de estudos que avaliem o impacto de seu uso na prática clínica.

Mais recentemente, na década de 90, foi desenvolvido o Opcrit por McGuffin et al na Inglaterra. ${ }^{12}$ Constitui um sistema diagnóstico computadorizado, composto por uma lista de 90 sintomas, capaz de fornecer diagnósticos de transtornos psicóticos segundo 11 critérios operacionais.

Como pode-se observar na Tabela 2, somente o DTREE e o Opcrit passaram por uma validação adequada com índices aceitáveis e foram implementados para uso clínico ou de pesquisa.

O uso de sistemas diagnósticos computadorizados, que empregam múltiplos critérios operacionais, mostra que o diagnóstico de esquizofrenia varia amplamente dependendo do critério utilizado. Bell et al avaliaram 479 pacientes com primeiro episódio psicótico, em 11 critérios diagnósticos diferentes, e verificaram que apenas $4,6 \%$ dos pacientes não foram classificados como esquizofrênicos em pelo menos um dos critérios diagnósticos, e que $1,7 \%$ foram classificados como esquizofrênicos em todos os sistemas diagnósticos. ${ }^{13}$

\section{Conclusões}

O emprego das técnicas de inteligência artificial na medicina e, mais recentemente, na psiquiatria, tem se mostrado promissor com o desenvolvimento de sistemas inteligentes que auxiliam na tomada de decisão no contexto clínico e no estudo de critérios diagnósticos.

Embora a literatura aponte para os benefícios na prática clínica com o uso de tais sistemas, a metodologia utilizada para a sua construção e validação precisa ser revista e aperfeiçoada. ${ }^{2,14-15}$ As perspectivas futuras concentram-se em desenvolver metodologias adequadas para o manejo de grandes quantidades de conhecimento, em aperfeiçoar as técnicas de aquisição de conhecimento e em construir sistemas portáteis e eficientes adequados ao contexto do usuário final.

\begin{tabular}{|c|c|c|c|c|}
\hline Diagno $^{8}(1968)$ & APA & $\operatorname{Sim}(k=0,59)$ & Não & Baixa performance \\
\hline Headmed $^{1}(1978)$ & empíricos & Não & Não & Pouco operacional \\
\hline Overseer $^{10}(1987)$ & $\begin{array}{l}\text { Protocolos de tratamento de } \\
\text { transtornos afetivos e psicóticos }\end{array}$ & Sim & Não & Baixa aceitação pelos usuários \\
\hline DTREE $^{11}(1988)$ & DSMIII-R, DSM-IV & $\operatorname{Sim}(k=0,80)$ & Sim & $\begin{array}{l}\text { Validado contra painel de especialistas para } \\
\text { esquizofrenia }\end{array}$ \\
\hline $\mathrm{OPCRIT}^{12}(1991)$ & 11 critérios operacionais & $\begin{array}{l}\text { Sim } \\
(k=0,85 \text { DSMIII-R; } \\
\text { k=0,72 RDC) }\end{array}$ & Sim & $\begin{array}{l}\text { Requer julgamento clínico e treinamento do } \\
\text { usuário }\end{array}$ \\
\hline
\end{tabular}




\section{Referências}

1. Ohayon MM. Utilisation des systèmes expert en psychiatrie. Rev Can Psychiatrie 1993;38(3):203-11.

2. Haynes RB, Walker CJ. Computer-aided quality assurance: a critical appraisal. Arch Intern Med 1987;147:1297-1301.

3. Walton RT, Dovey S, Harvey E, Freemantle N. Computer suport for determining drug dose: systematic review and meta-analysis. BMJ 1999;318:984-90.

4. Morelli RA, Bronzino JD, Goethe JW. Expert systems in Psychiatry: a review. J Med Syst 1987;11(213):157-68.

5. Yee WK. Knowledge coupling: support for psychiatric decision making. In: Miller MJ, Hammond KW, Hile MG, editors. Mental Health Computing. New York: Springer/Verlag; 1996. p. 389-414.

6. Smith R. What clinical information do doctors need? BMJ 1996;7064:313.

7. Slawson DC, Shaughnessy AF. Obtaining useful information from expert based sources. BMJ 1997;314:947.

8. Spitzer RL, Endicott J. DIAGNO: a computer program for psychiatric diagnosis utilizing the differential diagnostic procedure. Arch Gen Psychiatry 1968;18:747-55.
9. Williams TA, Johnson JH, Bliss EL. A computer assisted psychiatric assessment unit. Am J Psychiatry 1975;132(10):1074-6.

10.Bronzino JD, Morelli RA, Goeth JW. Overseer: a prototype expert system for monitoring drug treatment in the psychiatric clinic. IEEE Trans Biomed Eng 1989;36(5):533-40.

11. First MB, Opler LA, Hamilton RM, Linder J, Linfield LS, Silver $\mathrm{JM}$ et al. Evaluation in a inpatient setting of DTREE, a computerassisted diagnostic assessment procedure. Compr Psychiatry 1993;34(3):171-75

12.McGuffin P, Farmer A, Harvey I. A polydiagnostic application of operational criteria in studies of psychotic illness: development and reliability of the OPCRIT system. Arch Gen Psychiatry 1991;48:764-70.

13.Bell RC, Dudgeon P, McGorry PD, Jackson, HJ. The dimensionality of schizophrenia concepts in first-episode psychosis. Acta Psychiatr Scand 1998;97:334-42.

14.Wyatt J, Spiegelhalter D. Evaluating medical expert systems: what to test and how? Med Inform (Lond) 1990;15(3):205-17.

15.Lundsgaarde HP. Evaluating Medical expert systems. Soc Sci Med 1987;24(10):805-19.

\section{Correspondência: Denise Razzouk}

Doutoranda do Programa de Pós-graduação do Departamento de Psiquiatria da Unifesp/EPM

Rua Dr. Bacelar, 334, Vila Clementino - CEP 04026-001, São Paulo, SP

Fone/fax: (0xx11) 5084-7060 -E-mail:razzouk@psiquiatria.epm.br 